\title{
Molecules in Action: Extragalactic ISM at high resolution
}

\author{
Sergio Martín ${ }^{1,2}$ \\ ${ }^{1}$ European Southern Observatory, \\ Alonso de Córdova 3107, Vitacura, Santiago, Chile \\ email: smartin@eso.org \\ ${ }^{2}$ Joint ALMA Observatory, \\ Alonso de Córdova 3107, Vitacura, Santiago, Chile
}

\begin{abstract}
During the last two decade, observations have shown the potential of molecular tracers to get insights into the physical processes taking place in the central regions of active galaxies. However, observations were severely limited by both sensitivity and resolution. This resulted also in a limited sample of bright enough galaxies where molecular species other than carbon monoxide could be observed. Current instruments like ALMA and the upcoming NOEMA are already changing our view of the extragalactic ISM molecular observations. In fact, it is now possible to study the physical properties of individual spatially resolved star forming GMCs in external galaxies, as well as resolving the physical structure of the ISM in the surroundings of AGNs at scales of a few parsecs. Here I quickly review some of the most recent observational studies in the nuclear regions of galaxies which are setting new standards in the ways we can study the extragalactic ISM properties.
\end{abstract}

Keywords. ISM: abundances, ISM: molecules, galaxies: abundances, galaxies: active, galaxies: ISM, galaxies: seyfert, galaxies: starburst

\section{Introduction: Craving high resolution}

Almost a decade ago, the first unbiased spectral line survey was carried out towards the starburst galaxy NGC 253 (Martín et al. 2006) with the aim of exploring the limits of the observable chemical complexity in the extragalactic ISM. This work showed that increasing the number of observed species allowed direct comparison with Galactic environments which could provide clues on the overall leading heating mechanism in the central regions of galaxies. Also driven by the technical developments in available instantaneous bandwidth of $\mathrm{mm}$ and submm telescopes, a large number of spectral line surveys were carried out towards the brightest nearby active galaxies (see review by Martín 2011). The spectral line survey towards the starburst M 82 clearly showed that when compared to that of NGC 253, even at the large spatial scales sampled by single dish observations, the evident molecular abundance variations could be linked to the evolutionary state of the nuclear starburst (Aladro et al. 2011). However such molecular variations were not so evident when trying to establish a comparative chemical composition towards AGN dominated galaxies or towards luminous and ultra luminous galaxies through deep wide band spectral observations (Costagliola et al. 2011, 2015; Aladro et al. 2013, 2015; Martín et al. 2011).

Very deep single dish observations are able to increment our knowledge about the overall molecular composition of the extragalactic ISM. Such is the case of the recent tentative detection of $\mathrm{HC}_{5} \mathrm{~N}$ towards NGC 253 and NGC 4418 (Aladro et al. 2015; Costagliola et al. 2015). However, the sampled regions (larger than a few hundred parsecs) severely hinders the possibility of establishing a direct link between the enhancement of a given species 
and the type of galactic activity and therefore the diagnostic potential of molecular observations.

A number of pioneering interferometric studies resulted in compelling imaging of the extragalactic ISM in molecular lines other than carbon monoxide, the "universal" molecular gas tracer (García-Burillo et al. 2000, 2001, 2002, 2010; Usero et al. 2007; Meier \& Turner 2005, 2012). Though this studies showed the potential of $\mathrm{mm}$ and submm spectral observations to dissect the ISM through the enhanced emission selected molecular species, the number of available species was limited by sensitivity. This sensitivity issue biased chemical studies in galaxies towards the brightest objects in the sky.

\section{Starburst and AGN molecular observations at high resolution}

As a result of the observations carried out during its Cycle 0, the kickoff of ALMA, even at a fraction of its full potential, has shown to be a game changer also in the field of extragalactic astrochemistry. The ALMA observations towards NGC 253 represents an excellent example. Though originally aiming at the mapping of $\mathrm{CO}$ and the dense gas tracers $\mathrm{HCN}$ and $\mathrm{HCO}^{+}$(Leroy et al. 2015) the high sensitivity and large available bandwidth resulted in the detection and identification of 24 extra molecular transitions as well as some unidentified features (Meier et al. 2015). Though the images were probing scales similar to previous extragalactic works (50-100 pc), the unprecedented sensitivity allowed the imaging of faint transitions such as the rarer isotopologue of carbon monoxide, $\mathrm{C}^{17} \mathrm{O}$. The multi molecular imaging allowed to sketch a map of the different heating mechanisms in play within the central molecular zone of NGC 253. SiO and HNCO tracing the shocked molecular gas orbiting the nuclear region, with the latter disappearing with increasing $\mathrm{PDR}$ contribution towards center as determined from the $\mathrm{CN} / \mathrm{C}^{17} \mathrm{O}$ and $\mathrm{C}_{2} \mathrm{H} / \mathrm{H}^{13} \mathrm{CN}$ line ratios. The dense gas traced by $\mathrm{HCN}, \mathrm{HCO}^{+}$and $\mathrm{CN}$ located within the galactic plane at the base of the molecular outflow observed in CO (Bolatto et al. 2013) and the hydrogen recombination lines tracing the star formation right at the very inner regions (Bendo et al. 2015).

However, an even more compelling case is that of the direct imaging of the circumnuclear disk around the supermassive black holes in the nuclei of AGN galaxies. Not only it has become possible the multi molecular observation towards the brightest nearby Seyfert 2 luminous AGN, the galaxy NGC 1068 (Takano et al. 2014; Nakajima et al. 2015) but also fainter objects such as the galaxy NGC 1097 (Izumi et al. 2013; Martín et al. 2015) which is more than an order of magnitude fainter in X-ray and IR luminosity, as well as in molecular gas. Despite the different luminosities of their AGNs, as shown in Fig. 1 the distribution of species in the CND of both galaxies is remarkably similar, which suggests that the chemistry that we are observing is not directly driven by the X-ray radiation from the AGN. Within the central $\sim 100 \mathrm{pc}$ around the AGN species efficiently formed in the surface of dust grains, such as $\mathrm{HNCO}$ and $\mathrm{CH}_{3} \mathrm{OH}$, clearly delineate the circumnuclear disk. Other molecules like the shock tracer $\mathrm{SiO}$ or even more complex ones like cyanoacetylene $\left(\mathrm{HC}_{3} \mathrm{~N}\right)$ and acetonitrile $\left(\mathrm{CH}_{3} \mathrm{CN}\right)$ are observed to be spatially unresolved at the current resolution towards the very nuclear region. The compactness of the emission of these species shows the existence of molecular gas clumps at very close distances from the supermassive black hole which must dense enough to be able to protect these complex organic molecules from the pervasive X-ray dissociating radiation. The presence of $\mathrm{SiO}$ at such close distance from the AGN lead to think of its possible enhancement due to X-ray chemistry rather than just tracing shock material (García-Burillo et al. 2010). Actually, the achieved resolution is already close or at least comparable to that of existing chemical models of the the ISM chemistry in molecular 


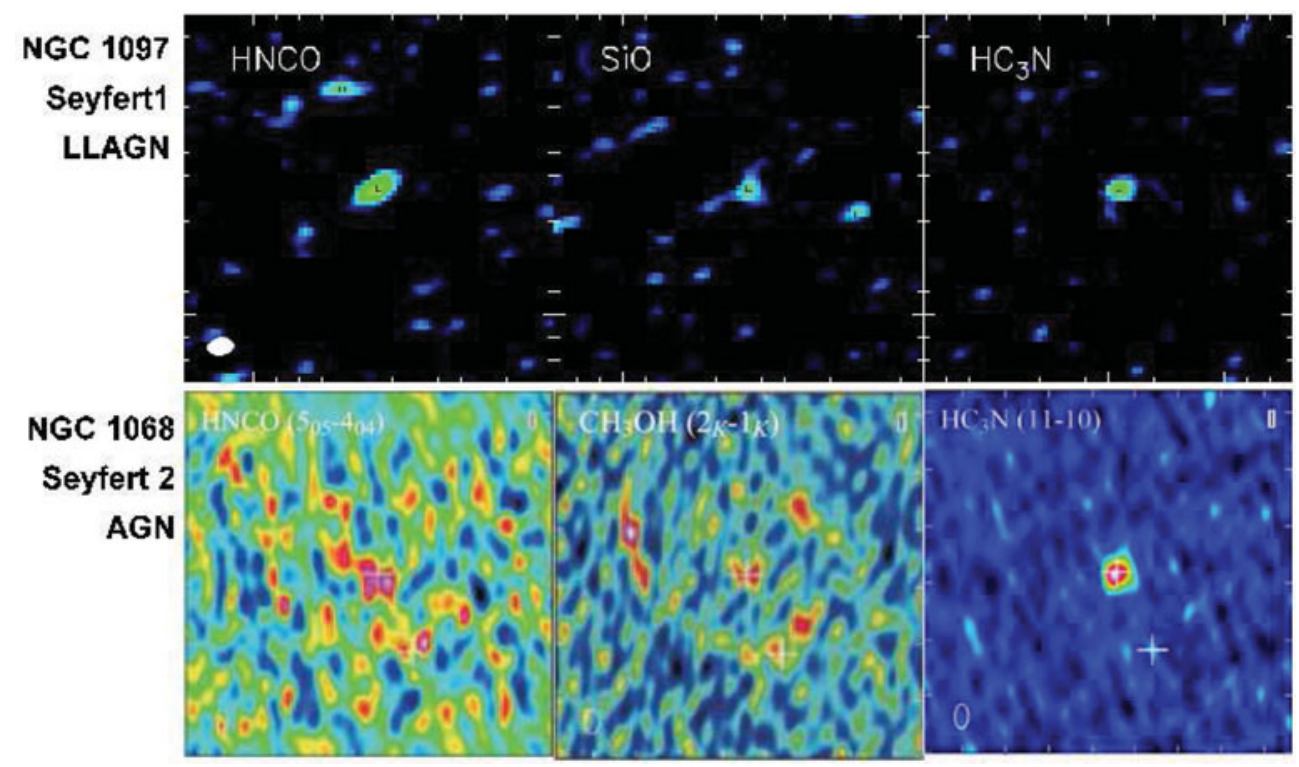

Figure 1. Multi-molecular observations within the inner $\sim 100 \mathrm{pc}$ of the low luminosity Sy 1 NGC 1097, and the bright Sy 2 nucleus in NGC 1068. Molecular observation show that even though there is an order of magnitude difference in luminosity between both nuclei, the ISM structure around the AGN show strong similarities. $\mathrm{HNCO}$ and $\mathrm{CH}_{3} \mathrm{OH}$ trace well the dense $\mathrm{CND}$ while $\mathrm{SiO}$ and $\mathrm{HC}_{3} \mathrm{~N}$ are unresolved and emitting within the very nucleus. Dense gas clumps must exist within the central tens of parsec in order to shield species such as $\mathrm{HC}_{3} \mathrm{~N}$ and $\mathrm{CH}_{3} \mathrm{CN}$ from the dissociation. Composite figure extracted from Martín et al. (2015) and Takano et al. (2014).

disks surrounding AGNs (Harada et al. 2013). Indeed, these models are able to reproduce such large abundances of $\mathrm{HC}_{3} \mathrm{~N}$ within $10-20 \mathrm{pc}$ from the AGN. The comparison of such models with even higher resolution multi-molecule imaging will impose important constraints in the physical conditions of the ISM around the AGN.

\section{3. $\mathrm{HCN} / \mathrm{HCO}^{+} / \mathrm{CS}$ as AGN-vs-SB diagnostic tools}

The search for key molecular fingerprints which would allow the creation of AGN-vsSB diagnostic diagrams has been around for more than a decade now. The pioneering work by Kohno et al. (2001) followed by multi-transition excitation studies by Krips et al. (2008) showed that the differences observed in the $\mathrm{HCN} / \mathrm{HCO}^{+}$ratio was actually the result of abundance differences and therefore might be a good probe to differentiate between AGN and SB dominated galaxies. This differentiation was supported by chemical modeling which could reproduce the overproduction of $\mathrm{HCN}$ in X-ray dominated regions (i.e. Meijerink et al. 2007). However the support from models was marginal since such $\mathrm{HCN} / \mathrm{HCO}^{+}$ratio enhancement was only above unity for relatively unattenuated gas in the surroundings of molecular clouds, which may not be representative of the dense gas medium around AGNs (Izumi et al. 2013).

Still, the differentiation based on this line ratio seems to be more prominent when using high resolution observations. The diagnostic diagram using the $\mathrm{HCN} / \mathrm{HCO}^{+}$in the high-J $4-3$ transitions together with the HCN/CS ratio (Izumi et al. 2013) shows such ratios in AGN dominated galaxies to be a factor $\sim 4$ higher than in SB dominated galaxies. The wider sample of observations explored by Izumi et al. (2015, submitted) 
shows clear evidence of the enhancement of these two ratios in the presence of an AGN. However, the high resolution observations of the $\mathrm{HCN} / \mathrm{HCO}^{+}$towards both $\mathrm{NGC} 1068$ and NGC 1097 (García-Burillo et al. 2014; Martín et al. 2015) proved that the highest HCN emission was not originated at the central few tens of parsec of the AGN but rather in the surrounding CND.

It has been claimed that the enhancement of $\mathrm{HCN}$ in the surroundings of the AGNs is actually related to the production of $\mathrm{HCN}$ via the endothermic hydrogenation reaction of $\mathrm{CN}$

$$
\mathrm{CN}+\mathrm{H}_{2} \rightarrow \mathrm{HCN}+\mathrm{H}, \gamma=820 \mathrm{~K}
$$

where $\gamma$ is the reaction barrier. Modeling of the chemistry of molecular clouds in the vicinity of an $\mathrm{AGN}$ shows how, irrespective of variations in the $\mathrm{X}$-ray ionization rate by several orders of magnitude, the HCN fractional abundance does not show strong variations. However, increasing the temperature of the gas between 100 and $800 \mathrm{~K}$ results in a dramatic HCN abundance enhancement (Harada et al. 2010, 2013; Izumi et al. 2013). Given that the heating due to the AGN would not be enough in low luminosity AGN such as the one in NGC 1097, hot gas chemistry likely induced by mechanical heating due to AGN jets and/or outflows could be the responsible for the observed abundances in the central regions of AGN dominated galaxies (Loenen et al. 2008; Aalto et al. 2012; Izumi et al. 2013; Martín et al. 2015). This mechanical heating could also be linked to the higher abundances of $\mathrm{SiO}$ at the very central region around the supermassive black hole.

\section{Molecular probes of compact obscured nuclei}

Molecular emission in the mm and submm has been claimed to be an excellent probe of the nuclei of dust obscured galaxies as these wavelengths are barely affected by obscuration. However, that might not apply to the central regions of compact obscured galaxies $(\mathrm{CON})$.

CONs could be defined as luminous and ultra-luminous infrared galaxies, radiating most of their energy as thermal dust emission in the IR, with very compact (few tens of parsec) Compton thick nuclei. These objects might be the dominant population among the most luminous extragalactic objects, but still, reaching their inner regions may be an observational challenge even at millimeter wavelengths.

Some examples of this kind of objects would be the LIRG NGC 4418 (i.e. Costagliola et al. 2015) or the nearest ULIRG, Arp 220. Though the archetypical Arp 220 has been studied for decades at all possible wavelengths, its two nuclei keep surprising us when going to the highest resolutions. Recent interferometric observations with the SMA and $\mathrm{PdBI}$ resulted in the detection of P-cygni profiles in the high density tracers $\mathrm{HCO}^{+}$and $\mathrm{SiO}$ towards both nuclei (Sakamoto et al. 2009; Tunnard et al. 2015) which has been interpreted as $\sim 100 \mathrm{~km} \mathrm{~s}^{-1}$ molecular gas outward motions. During the ALMA Cycle 0 of observations, a number of projects have also aimed to peer into the nuclei of Arp 220. Wilson et al. (2014) imaged the dust continuum at $690 \mathrm{GHz}$ deriving dust temperatures of 80 and $200 \mathrm{~K}$ for the eastern and western nucleus, respectively. The kinematics of the molecular gas around the nuclei was studied via a fit of a simple disk model to the dense gas emission traced by HCN (Scoville et al. 2015). However opacity towards the nuclei does play a crucial role in the observed line profiles and needs to be taken into account for any study of Arp 220. Such is the case of the work presented by Rangwala et al. (2015) where they modeled the CO 6-5 kinematics of the eastern nucleus with a highly turbulent dis with high line center opacity and a large temperature gradient to account 


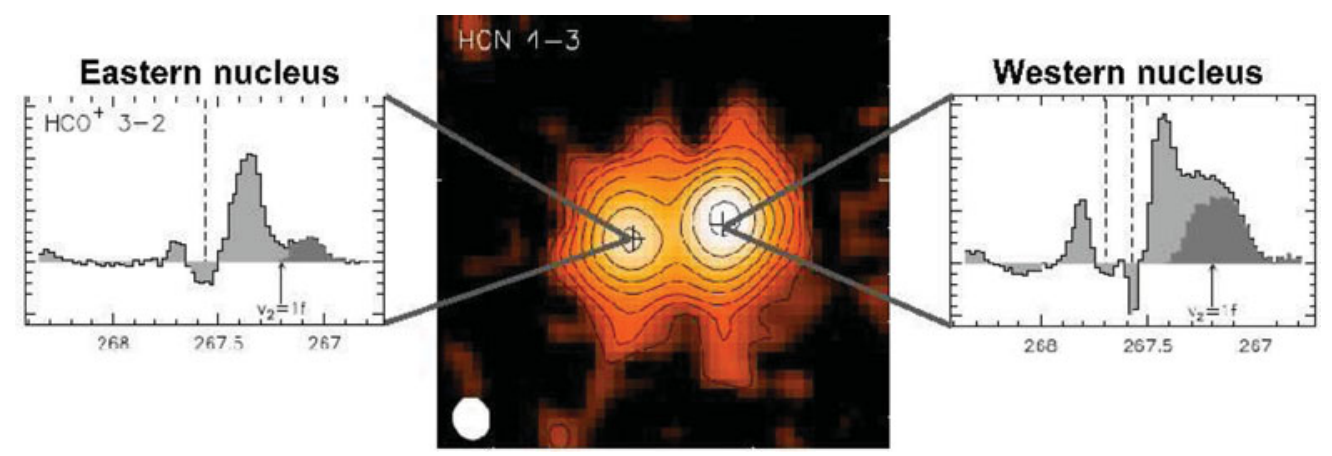

Figure 2. Integrated emission of $\mathrm{HCN} J=4-3$ transition towards the two nuclei of Arp 220 at a resolution of $\sim 0.5^{\prime \prime}$. The $\mathrm{HCO}^{+} J=3-2$ spectra extracted from both nuclei are shown. Heavy absorption systems account for $\sim 70 \%$ of the emission preventing the direct observation of the central few tens of parsecs around the nuclei. Dark grey histograms show the strong contribution from the vibrationally excited $\left(v_{2}=1\right)$ transitions of $\mathrm{HCN}$ to the $\mathrm{HCO}^{+}$profiles. This vibrationally excited emission is likely the best probe of the inner hot regions around their nuclear power sources. At the current resolution this source might be either an AGN or a hot compact starburst.

for the central observed absorption. The various absorption system towards the Western nucleus (Martín et al. 2015 submitted) makes it more difficult to accurately model its kinematics.

Arp 220 showed an extremely prolific spectrum in the frequency scan carried out with the SMA at $1.3 \mathrm{~mm}$ (Martín et al. 2011). In these observations most of the emission lines showed a double peak profile which had been classically considered as two molecular components resulting from the distinct kinematics of the two nuclei. However, recent observations have shown that absorption close to the systemic velocity appears to be severely affecting the molecular emission in Arp 220 towards the central few tens of parsec. The recently completed spectral line scan at $0.65^{\prime \prime}$ resolution with ALMA toward Arp 220, covering frequencies between 210 and $360 \mathrm{GHz}$, results again in a mind-blowing molecular complexity towards both nuclei with dozens of molecules identified and hundreds of transitions blended in a confusion limited spectrum.

All the standard tracers of molecular gas, namely $\mathrm{CO}, \mathrm{HCN}, \mathrm{HCO}^{+}, \mathrm{HNC}$ and $\mathrm{CS}$, show absorbed profiles in the direction of both Arp 220 nuclei. Either due to selfabsorption or continuum absorption, these tracers do not allow us to peer into the central molecular zone around the two nuclei. Fig. 2 shows how approximately $70 \%$ of the line is absorbed towards both nuclei. This fact, which is likely extended to most CONs, does prevent an accurate measurement of either the overall molecular gas content through $\mathrm{CO}$ or the dense gas content via the classical dense gas tracers. This absorption effect has implications in the conclusions from KennicuttSchmidt laws where the molecular gas content is derived from absorbed profiles. The study by Graciá-Carpio et al. (2008) in a sample of LIRGs and ULIRGs resulted in an enhancement of the star formation efficiency (SFE) of the dense gas towards the higher end of luminosities of the sampled galaxies. However, the dense gas content based on HCN $1-0$ observations might also affected by absorption, similar to what is observed towards Arp 220. Indeed, some of the higher signal to noise detections in this work showed double peak profiles which would support this absorption scenario. The estimate of the dense gas content in this galaxies would therefore be underestimated, leading to an overestimate of the SFE. 


\section{The ultimate tracer: Vibrationally excited emission lines}

Apart from the difficulty of estimating the amount of both low and high density molecular gas in compact obscured nuclei, it still remains the question on how to actually observe their very inner regions affected by both obscuration and absorption. It appears that the answer might be actually in the vibrational levels of the commonly observed dense gas tracers, as shown in Fig. 2.

The first vibrational transition of HCN was detected in absorption towards Arp 220 in radio wavelengths (Salter et al. 2008). Only during the last five years that a number of detections of vibrationally excited $\mathrm{HCN}$ and $\mathrm{HC}_{3} \mathrm{~N}$ have been reported towards the LIRG NGC 4418 (Sakamoto et al. 2010; Costagliola et al. 2013, 2015), and the ULIRGs Arp 220 (Martín et al. 2011) and IRAS 20551-4250 (Imanishi \& Nakanishi 2013). Indeed vibrationally excited emission has only been detected towards luminous infrared galaxies in the extragalactic ISM. In the case of $\mathrm{HC}_{3} \mathrm{~N}$, the vibrational emission is one of the main polluters in the mm and submillimeter spectrum of NGC 4418 and Arp 220 (Martín et al. 2011; Costagliola et al. 2015), with groups of transitions every $\sim 9 \mathrm{GHz}$.

The vibrational transitions of HCN are separated by $\sim 1000 \mathrm{~K}$ from the ground level and cannot be collisionally excited at such high observed intensity (Sakamoto et al. 2010; Imanishi \& Nakanishi 2013). They can however be efficiently excited through infrared pumping provided a bright source of IR emission $(T(14 \mu \mathrm{m})>100 \mathrm{~K}$ : Aalto et al. 2015) in the nuclei of these galaxies. Thus the detection of vibrationally excited emission requires the presence of a very hot nucleus with a temperature gradient out to the cooler surrounding envelope which would be responsible for the observed absorption in all tracers. This idea is supported by the study of the excitation of the vibrational transitions of $\mathrm{HC}_{3} \mathrm{~N}$ which yields high excitation temperatures $>300 \mathrm{~K}$ in both NGC 4418 and Arp 220.

The largest sample of galaxies with vibrationally excited HCN emission detected, consisting on 8 luminous IR galaxies, was recently compiled by Aalto et al. (2015). This study tentatively showed an correlation between the $L_{\mathrm{vib}} / \mathrm{L}_{\mathrm{FIR}}$ ratio and the velocity of the observed outflows in the sampled galaxies, with the highest vibrational emission luminosity in sources where no outflow was previously detected. This fact has been claimed as a possible trace of an early stage of evolution of their nuclear power source. Whether this powering source is an AGN or a hot compact starburst will require higher resolution observations.

In the case of Arp 220, evidences are put forth that suggest the presence of an obscured AGN in its Western nucleus (see Downes \& Eckart 2007, and references therein). The heating due to the supermassive black hole might be at the origin of the vibrationally excited emission. Higher resolution observations of these transitions will finally reveal whether an AGN is the source of the huge IR luminosity of Arp 220 and whether both nuclei harbor such hidden accreting supermassive black holes.

\section{References}

Aalto, S., Garcia-Burillo, S., Muller, S., et al. 2012, A\&A, 537, A44

Aalto, S., Martin, S., Costagliola, F., et al. 2015, arXiv1504.06824

Aladro, R., Martín, S., Martín-Pintado, J., et al. 2011, A\&A, 535, A84

Aladro, R., Martín, S., Riquelme, D., et al. 2015, arXiv1504.03743

Aladro, R., Viti, S., Bayet, E., et al. 2013, A\&A, 549, A39

Bendo, G. J., Beswick, R. J., D'Cruze, M. J., et al. 2015, MNRAS, 450, L80

Bolatto, A. D., Warren, S. R., Leroy, A. K., et al. 2013, Nature, 499, 450

Costagliola, F., Aalto, S., Rodriguez, M. I., et al. 2011, A\& A, 528, A30 
Costagliola, F., Aalto, S., Sakamoto, K., et al. 2013, A $\mathscr{E} A$, 556, A66

Costagliola, F., Sakamoto, K., Muller, S., et al. 2015, arXiv1506.09027

Downes, D. \& Eckart, A. 2007, A\&BA, 468, L57

García-Burillo, S., Combes, F., Usero, A., et al. 2014, A\&3A, 567, A125

García-Burillo, S., Martín-Pintado, J., Fuente, A., \& Neri, R. 2000, A\&3A, 355, 499

García-Burillo, S., Martín-Pintado, J., Fuente, A., \& Neri, R. 2001, ApJ, 563, L27

García-Burillo, S., Martín-Pintado, J., Fuente, A., Usero, A., \& Neri, R. 2002, ApJ, 575, L55

García-Burillo, S., Usero, A., Fuente, A., et al. 2010, A\&A, 519, A2

Graciá-Carpio, J., García-Burillo, S., Planesas, P., Fuente, A., \& Usero, A. 2008, A 6 A, 479, 703

Harada, N., Herbst, E., \& Wakelam, V. 2010, ApJ, 721, 1570

Harada, N., Thompson, T. A., \& Herbst, E. 2013, ApJ, 765, 108

Imanishi, M. \& Nakanishi, K. 2013, $A J, 146,91$

Izumi, T., Kohno, K., Martín, S., et al. 2013, PASJ, 65, 100

Kohno, K., Matsushita, S., Vila-Vilaró, B., et al. 2001, in J. H. Knapen, J. E. Beckman, I. Shlosman, \& T. J. Mahoney (eds.), The Central Kiloparsec of Starbursts and AGN: The La Palma Connection, ASP-CS, No. 249, p. 672

Krips, M., Neri, R., García-Burillo, S., et al. 2008, ApJ, 677, 262

Leroy, A. K., Bolatto, A. D., Ostriker, E. C., et al. 2015, ApJ, 801, 25

Loenen, A. F., Spaans, M., Baan, W. A., \& Meijerink, R. 2008, A $6 A$, 488, L5

Martín, S. 2011, in J. Cernicharo \& R. Bachiller (eds.), The Molecular Universe, Proc. IAU Symposium No. 280, p. 351

Martín, S., Kohno, K., Izumi, T., et al. 2015, A\&\&A, 573, A116

Martín, S., Krips, M., Martín-Pintado, J., et al. 2011, A\&A, 527, A36

Martín, S., Mauersberger, R., Martín-Pintado, J., Henkel, C., \& García-Burillo, S. 2006, ApJS, 164,450

Meier, D. S. \& Turner, J. L. 2005, ApJ, 618, 259

Meier, D. S. \& Turner, J. L. 2012, ApJ, 755, 104

Meier, D. S., Walter, F., Bolatto, A. D., et al. 2015, ApJ, 801, 63

Meijerink, R., Spaans, M., \& Israel, F. P. 2007, A\& $A$, 461, 793

Nakajima, T., Takano, S., Kohno, K., et al. 2015, PASJ, 67, 8

Rangwala, N., Maloney, P., Wilson, C., et al. 2015, ApJ, 806, 17

Sakamoto, K., Aalto, S., Evans, A. S., Wiedner, M. C., \& Wilner, D. J. 2010, ApJ, 725, L228

Sakamoto, K., Aalto, S., Wilner, D. J., et al. 2009, ApJ, 700, L104

Salter, C. J., Ghosh, T., Catinella, B., et al. 2008, AJ, 136, 389

Scoville, N., Sheth, K., Walter, F., et al. 2015, ApJ, 800, 70

Takano, S., Nakajima, T., Kohno, K., et al. 2014, PASJ, 66, 75

Tunnard, R., Greve, T. R., Garcia-Burillo, S., et al. 2015, ApJ, 800, 25

Usero, A., García-Burillo, S., Martín-Pintado, J., Fuente, A., \& Neri, R. 2007, New Astron. Revs, 51, 75

Wilson, C. D., Rangwala, N., Glenn, J., et al. 2014, ApJ, 789, L36 